\title{
THE FAMILY APPROACH TO TOTAL COCOMPLETENESS AND TOPOSES
}

\author{
BY
}

\author{
ROSS STREET
}

\begin{abstract}
A category with small homsets is called total when its Yoneda embedding has a left adjoint; when the left adjoint preserves pullbacks, the category is called lex total. Total categories are characterized in this paper in terms of special limits and colimits which exist therein, and lex-total categories are distinguished as those which satisfy further exactness conditions. The limits involved are finite limits and intersections of all families of subobjects. The colimits are quotients of certain relations (called congruences) on families of objects (not just single objects). Just as an arrow leads to an equivalence relation on its source, a family of arrows into a given object leads to a congruence on the family of sources; in the lex-total case all congruences arise in this way and their quotients are stable under pullback. The connection with toposes is examined.
\end{abstract}

In the introduction to [7] and elsewhere, I have maintained that a Grothendieck topos [1] is a Barr exact category [2] for which the exactness axioms are extended to deal with families of arrows and not just single arrows. The present paper makes this explicit without the bicategorical paraphernalia. The concepts involved are far-reaching and the main result (Theorem 14) characterizes both total categories [8] and lex-total categories $[\mathbf{8}, \mathbf{6}]$ in familial terms. Total categories are precisely the categories, algebraic and topological $[\mathbf{1 2}, \mathbf{1 0}]$, at which traditional category theory was aimed. Lex-total categories are essentially toposes [6].

Recall that a category is regular when finite limits exist, every arrow factors as a strong epic arrow followed by a monic arrow, and every pullback of each strong epic arrow is strong epic. (Strong epics are defined from the monics by a diagonal property [5].) That this agrees with the definition of Barr [2] when finite limits exist is an observation of Joyal [9]. I am indebted to Max Kelly for providing a proof of Joyal's observation, on which proof the present family version (Theorem 3 ) is based.

The family version of a regular category, a familially regular category, takes the notion of "strong epic family of arrows" as basic, requires that families of arrows should factor through strong epic families via monics (this already implies the existence of many limits including all finite ones; see Proposition 1), and requires strong epic families to be stable under pullback.

Received by the editors May 10, 1983 and, in revised form, September 21, 1983.

1980 Mathematics Subject Classification. Primary 18A30, 18A32, 18B25; Secondary 18F10, 18F20.

Key words and phrases. Total and lex-total category, exact category, factorization of families, Grothendieck topos, finitely presentable, universal extremal epimorphic family.

(C)1984 American Mathematical Society $0002-9947 / 84 \$ 1.00+\$ .25$ per page 
A category is exact when it is regular and every equivalence relation is a kernel pair. The familial version of this is more subtle and requires understanding what should be meant by the kernel of a family of arrows with the same target and what should be meant by an equivalence relation on a family of objects. Even then not every equivalence relation should be a kernel, only those which satisfy a certain size condition; I call them congruences. Congruences can be viewed as diagrams in the category; the coprojections for the colimits of such diagrams give families of arrows called quotients; these colimits automatically exist in a familially exact category.

Quotients of congruences exist in total categories (although not all congruences are kernels in this case). In fact, this existence is the principal condition in the characterization of total categories (Theorem 14).

These ideas apply to categories with no size restriction on their sets of arrows, unlike totality and lex-totality which require small homsets. For categories with small homsets Theorem 14 asserts that familial exactness is equivalent to lex-totality. With a mild size restriction on a category, it is lex-total precisely when it is a Grothendieck topos (the proof of this, basically due to Peter Freyd, appears in [6]). I believe the family approach brings out the essence of topos theory in a way which unifies the divers aspects both logical and geometric.

In am grateful to Macquarie University and the Australian Research Grants Scheme (ARGS) for making possible my collaboration with Aurelio Carboni and Brian Day on projects intimately connected with this work.

1. Familial preliminaries. The category of small sets is denoted by $\mathscr{S}$.

Let $A$ denote a category and let $a$ be an object of $A$. Let $\operatorname{Fam}(a)$ denote the ordered set of families $U=\left(\phi_{i}: u_{i} \rightarrow a\right)_{i \in I}$ of arrows into $a$ where $U \leqslant U^{\prime}$ means that each member $\phi_{i}$ of $U$ factors $\phi_{i}=\phi_{j}^{\prime} \theta$ through some member $\phi_{j}^{\prime}$ of $U^{\prime}$. Each arrow $\alpha: a \rightarrow b$ gives an order-preserving function $\alpha_{*}$ : $\operatorname{Fam}(a) \rightarrow \operatorname{Fam}(b)$ whose value at $U$ is $\alpha_{*} U=\left(\alpha \phi_{i}: u_{i} \rightarrow b\right)_{i \in I}$. There is a function $\alpha^{*}: \operatorname{Fam}(b) \rightarrow \operatorname{Fam}(a)$ which is right adjoint to $\alpha_{*}$. The value of $\alpha^{*}$ at $V=\left(\psi_{j}: v_{j} \rightarrow b\right)_{j \in J}$ is the family $\alpha^{*} V$ indexed by the set of triples $(j, \sigma, \tau)$, where $j \in J, \sigma: x \rightarrow a, \tau: x \rightarrow v_{j}$ with $\alpha \sigma=\psi_{j} \tau$, and the $(j, \sigma, \tau)$ th member of $\alpha^{*} V$ is $\sigma: x \rightarrow a$. It is easily verified that $U \leqslant \alpha^{*} V$ if and only if $\alpha_{*} U \leqslant V$.

Notice that, if $A$ has pullbacks, $\gamma^{*} V$ is isomorphic to the family of pullbacks of the members of $V$ along $\gamma$. So $\gamma^{*} V$ can be taken to have the same indexing set $J$ as $V$.

A family $U$ of arrows into $a$ is called epic when, for all $\alpha, \beta: a \rightarrow b$, if $\alpha_{*} U \cong \beta_{*} U$ then $\alpha=\beta$. The family $U$ is called strong epic if, furthermore, for all monics $\mu$ : $c \rightarrow b$, and arrows $\alpha: a \rightarrow b$, if every member of $\alpha_{*} U$ factors through $\mu$ then $\alpha$ factors through $\mu$. If $A$ has finite limits, a family $U$ into $a$ is strong epic if and only if $U \cong \mu_{*} V$ with $\mu$ monic implies $\mu$ invertible.

A family of arrows out of an object of $A$ is monic when it is epic as a family in $A^{\text {op }}$.

Suppose $\xi: J \rightarrow I$ is a function, $U=\left(\phi_{i}: u_{i} \rightarrow a\right)_{i \in I}$ is a family of arrows into $a$, and, for each $i \in I, V_{i}=\left(\psi_{i j}: v_{i j} \rightarrow u_{i}\right)_{j \in J_{i}}$ is a family of arrows into $u_{i}$ indexed by the fibre $J_{i}=\xi^{-1}(i)$ of $\xi$ over $i$. The family $U V=\left(\phi_{i} \psi_{i j}: v_{i j} \rightarrow a\right)_{\xi j=i}$ indexed by $J$ is called a composite family. Given that each $V_{i}$ is [strong] epic, the family $U V$ is [strong] epic if and only if $U$ is. 
Proposition 1. The following conditions on a category are equivalent:

(a) finite limits and intersections of arbitrary families of monics exist;

(b) finite products exist and each family $U$ of arrows into an object a has the form $\mu_{*} V$ where $\mu$ is a monic into $a$ and $V$ is a strong epic family;

(c) for all families $\left(u_{i}\right)_{i \in I},\left(a_{m}\right)_{m \in M}$ of objects where $M$ is finite, and, for all families $\left(\phi_{i}^{m}: u_{i} \rightarrow a_{m}\right)_{i \in I, m \in M}$ of arrows, there exist a strong epic family $\left(\psi_{i}: u_{i} \rightarrow c\right)_{i \in I}$ and $a$ monic family $\left(\alpha_{m}: c \rightarrow a_{m}\right)_{m \in M}$ such that $\alpha_{m} \psi_{i}=\phi_{i}^{m}$;

(d) as for (c) except that $M$ is restricted to having at most 2 elements.

Proof. (a) $\Rightarrow$ (b). Take $\mu: t \rightarrow a$ to be the intersection of all monics through which all members of $U$ factor. Then $U=\mu_{*} V$ for some family $V$. It remains to see that $V$ is strong epic. Since $A$ is finitely complete, we need only show that $V=\nu_{*} W$ with $\nu$ monic implies $\nu$ invertible. But $V=\nu_{*} W$ implies $U=(\mu \nu)_{*} V$; so $\mu \nu \leqslant \mu$ from the definition of $\nu$. So $\nu$ is invertible.

(b) $\Rightarrow$ (c). Apply (b) to the family $U$ of arrows $\left(\phi_{i}^{m}\right): u_{i} \rightarrow \prod_{m \in M} a_{m}$ indexed by $I$.

(c) $\Rightarrow$ (d). 0,1,2 are finite.

(d) $\Rightarrow$ (a). To obtain a terminal object apply (d) with $M$ empty, $I$ the set of all objects of $A$, and $u_{a}=a$. To construct a pullback for $\theta: a \rightarrow b, \theta^{\prime}: a^{\prime} \rightarrow b$, let $I$ denote the set of spans $i=\left(a \stackrel{\rho}{\leftarrow} r \stackrel{\rho^{\prime}}{\rightarrow} a^{\prime}\right)$ such that $\theta \rho=\theta^{\prime} \rho^{\prime}$. Apply (d) to the family $\left(\phi_{i}^{m}\right)_{i \in I, m \in M}$ where $M=2, \phi_{i}^{0}=\rho, \phi_{\alpha^{\prime}}^{1}=\rho^{\prime}$; this yields a (strong) epic family $\left(\psi_{i}: r \rightarrow c\right)_{i \in I}$ and a monic span $a \stackrel{\alpha}{\leftarrow} c \stackrel{\alpha^{\prime}}{\rightarrow} a^{\prime}$ such that $\alpha \psi_{i}=\rho, \alpha^{\prime} \psi_{i}=\rho^{\prime}$. Then $\alpha, \alpha^{\prime}$ provide a pullback for $\theta, \theta^{\prime}$.

Let $U=\left(\phi_{i}: u_{i} \rightarrow a\right)_{i \in I}$ be an arbitrary family of monics $\phi_{i}$ into $a$. Let $T$ be the family of arrows $\rho: r \rightarrow a$ which factor through all $\phi_{i}$. By (d) with $M=1, T=\mu_{*} V$ where $\mu: t \rightarrow a$ is monic and $V$ is strong epic. Since each member of $\mu_{*} V$ factors through the monic $\phi_{i}$ and $V$ is strong epic, $\mu$ factors through each $\phi_{i}$. So clearly, $t$ is the intersection of the $\phi_{i}, i \in I$.

2. Kernels and quotients. A span from $a$ to $b$ in the category $A$ is a pair $(\rho, \sigma)$ of arrows $\rho: r \rightarrow a, \sigma: r \rightarrow b$. A relation on a family $u=\left(u_{i}\right)_{i \in I}$ of objects $u_{i}$ of $A$ is a function $R$ which assigns to each $(i, j) \in I \times I$ a set $R_{j i}$ of spans from $u_{i}$ to $u_{j}$. A relation $R$ on $u$ is called saturated when each $R_{j i}$ is a crible of spans; that is, $(\rho, \sigma) \in R_{j i}$ implies $(\rho \theta, \sigma \theta) \in R_{j i}$ for all arrows $\theta$ for which the composite $\rho \theta$ is defined. Each set of spans generates a crible of spans and so each relation generates a saturated relation. Relations on $u$ are ordered by inclusion of their saturations.

The diagonal relation $\Delta$ on $u$ has $(\rho, \sigma) \in \Delta_{j i}$ if and only if $i=j$ and $\rho=\sigma$. This is already saturated. It is isomorphic to the relation which contains only the identity spans of the $u_{i}$.

Suppose $U=\left(\phi_{i}: u_{i} \rightarrow a\right)_{i \in I}$ is a family of arrows in $A$ with the same target $a$. The kernel of $U$ is the relation $E$ on $U=\left(u_{i}\right)_{i \in I}$ such that $(\rho, \sigma) \in E_{j i}$ when $\phi_{i} \rho=\phi_{j} \sigma$. If $A$ has pullbacks, the kernel of $U$ is isomorphic to the relation $R$ obtained by taking each $R_{j i}$ to be a singleton consisting of a pullback for $\phi_{i}, \phi_{j}$.

If $\mu: a \rightarrow b$ is monic then $\mu_{*} U$ and $U$ have the same kernel. Under certain conditions the converse can be proved for strong epic $U$. 
Proposition 2. Suppose $A$ has the property that, for all arrows $\alpha$, the function $\alpha^{*}$ takes strong epic families to epic families. Suppose $\mu: a \rightarrow b$ is an arrow and $U$ is $a$ strong epic family of arrows into a. If $U$ and $\mu_{*} U$ have the same kernel then $\mu$ is monic.

Proof. Suppose $\alpha, \beta: x \times a$ are such that $\mu \alpha=\mu \beta$. Put

$$
K=\left\{\left(i, \rho: r \rightarrow u_{i}, \sigma: r \rightarrow x\right) \mid \phi_{i} \rho=\alpha \sigma\right\}
$$

so that $\alpha^{*} U=(\sigma: r \rightarrow x)_{(i, \rho, \sigma) \in K}$ is an epic family. For each $(i, \rho, \sigma) \in K$, put

$$
L_{(i, \rho, \sigma)}=\left\{\left(j, \kappa: s \rightarrow r, \lambda: s \rightarrow u_{j}\right) \mid \beta \sigma \kappa=\phi_{j} \lambda\right\}
$$

so that $(\beta \sigma)^{*} U=(\kappa: s \rightarrow r)_{(j, \kappa, \lambda) \in L_{(i, \rho, \sigma)}}$ is an epic family. By composing we obtain an epic family $(\sigma \kappa: s \rightarrow x)_{(i, \rho, \sigma) \in K,(j, \kappa, \lambda) \in L_{(i, \rho, \sigma)}}$. So, in order to prove $\alpha=\beta$, it suffices to prove $\alpha \sigma \kappa=\beta \sigma \kappa$ for all $(i, \rho, \sigma) \in K,(j, \kappa, \lambda) \in L_{(i, \rho, \sigma)}$. Now $\mu \phi_{i} \rho \kappa=$ $\mu \alpha \sigma \kappa=\mu \beta \sigma \kappa=\mu \phi_{j} \lambda$. Since $\mu_{*} U, U$ have the same kernel, it follows that $\phi_{i} \rho \kappa=\phi_{j} \lambda$. Hence $\alpha \sigma \kappa=\beta \sigma \kappa$.

A quotient for a relation $R$ on $u=\left(u_{i}\right)_{i \in I}$ is a family $L=\left(\lambda_{i}: u_{i} \rightarrow c\right)_{i \in I}$ of arrows which displays $c$ as a colimit for the diagram as indicated below.

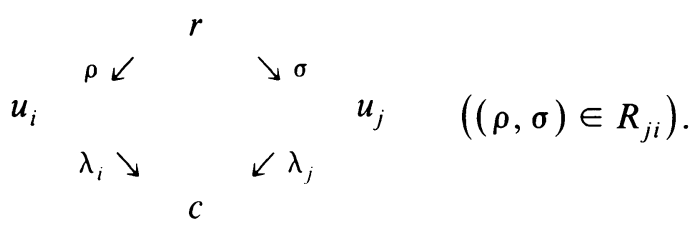

A quotient for $R$ is also a quotient for any relation isomorphic to $R$. If $L$ is a quotient for some relation then it is a quotient for its kernel. It is easy to see that quotients for relations are strong epic families; the next result gives conditions under which the converse holds.

Theorem 3. Suppose A satisfies the special case of Proposition 1(c) for which $M=2$, and suppose $A$ has the property in Proposition 2. Then every strong epic family is the quotient of its kernel.

Proof. Suppose $V=\left(\theta_{i}: u_{i} \rightarrow a\right)_{i \in I}$ is strong epic. Let $E$ be the kernel of $V$. To prove $V$ is a quotient for $E$ consider a family $\left(\psi_{i}: u_{i} \rightarrow b\right)_{i \in I}$ such that $\psi_{i} \rho=\psi_{j} \sigma$ whenever $(\rho, \sigma) \in E_{j i}$. By hypothesis, there exist a strong epic family $U=$ $\left(\phi_{i}: u_{i} \rightarrow x\right)_{i \in I}$ and a monic span $a \stackrel{\alpha}{\leftarrow} x \stackrel{\beta}{\rightarrow} b$ such that $\theta_{i}=\alpha \phi_{i}, \psi_{i}=\beta \psi_{i}$. Since $\left(\theta_{i}\right),\left(\phi_{i}\right)$ are strong epic, $\alpha$ is strong epic. Since $\alpha, \beta$ are jointly monic and $\phi_{i} \rho=\phi_{j} \sigma$ implies $\psi_{i} \rho=\psi_{j} \sigma$, the kernel of $U$ is $E$. By Proposition 2, $\alpha$ is monic. So $\alpha$ is invertible. So each $\psi_{i}$ factors through $\theta_{i}$ via $\beta \alpha^{-1}$.

A category $A$ is said to be familially regular when it satisfies the equivalent conditions of Proposition 1 and, for all arrows $\alpha$, the function $\alpha^{*}$ takes strong epic families to strong epic families.

COROLlaRY 4. In a familially regular category, every strong epic family is the quotient of its kernel. 
Recall that a set $G$ of objects of a category $A$ is called a strong generator for $A$ when, for all objects $a$ of $A$, there exists a strong epic family of arrows into $a$ whose members all have sources in $G$. This implies the condition that, for all $\alpha: a \rightarrow b$ in $A$, if $A(g, \alpha)$ is a bijection for all $g \in G$ then $\alpha$ is invertible; and when $A$ has pullbacks, the condition is equivalent to strong generation.

COROLlARY 5. In any category satisfying the hypotheses of Theorem 3 (in particular, a familially regular category), any strong generator is dense.

Proof. Suppose $G$ is a strong generator for $A$ and $\Theta_{g}: A(g, a) \rightarrow A(g, b)$ are functions natural in $g \in G$. Let $I$ be the set of arrows $\xi: g \rightarrow a$ with source in $G$. Let $U$ be the family whose $\xi$ th member is $\xi: g \rightarrow a$. Then $U$ is strong epic. By naturality of $\Theta$, we have that, for $\xi, \zeta \in I, \xi \rho=\zeta \sigma$ implies $\Theta_{g}(\xi)=\Theta_{h}(\zeta)$. By Theorem 3, there exists a unique $\alpha: a \rightarrow b$ such that $\alpha \xi=\Theta_{g}(\xi)$ for all $\xi \in I$. So $\Theta_{g}=A(g, \alpha)$. So $G$ is dense.

3. Congruences. It was observed in $\S 2$ that those relations which are kernels are saturated. In fact, such relations enjoy further properties which we shall now examine.

The natural context for these properties seems to be that of enriched categories with a bicategory as base. For a category $A$, the bicategory $\mathscr{W}_{A}$ of interest was used by Walters [11] and is defined as follows. The objects are the objects of $A$. The hom-category $\mathscr{W}_{A}(a, b)$ is the ordered set of cribles of spans from $a$ to $b$, ordered by inclusion. If $P: a \rightarrow b, Q: b \rightarrow c$ are cribles of spans then $Q \circ P: a \rightarrow c$ consists of those spans $(\rho, \tau)$ from $a$ to $c$ for which there exists $\sigma$ such that $(\rho, \sigma) \in P$, $(\sigma, \tau) \in Q$.

A category $E$ enriched in $\mathscr{W}_{A}$ (see $[11,3]$ ) consists of a set $I$ (of objects of $E$ ), a family $u$ of objects $u_{i}$ of $\mathscr{W}_{A}$ indexed by $i \in I$ (called the supporting family of $E$ ), and a family of arrows $E_{j i}: u_{i} \rightarrow u_{j}$ in $\mathscr{W}_{A}$ indexed by $(i, j) \in I \times I$, such that $E_{i i}$ contains the identity crible of $u_{i}$ and $E_{k j} \circ E_{j i} \leqslant E_{k i}$. Call $E$ symmetric when $(\rho, \sigma) \in E_{j i}$ if and only if $(\sigma, \rho) \in E_{i j}$.

In other words, a $\mathscr{W}_{A}$-enriched category $E$ with supporting family $u=\left(u_{i}\right)_{i \in I}$ is precisely a saturated relation on $u$ which is reflexive and transitive.

An equivalence relation on $u$ is a saturated, reflexive, transitive, symmetric relation $E$ on $u$ such that, for all spans $(\rho, \sigma)$ from $u_{i}$ to $u_{j}$ and all strong epic families $V=\left(\psi_{m}: v_{m} \rightarrow r\right)_{m \in M}$ into the source $r$ of $\rho, \sigma$, if $\left(\rho \psi_{m}, \sigma \psi_{m}\right) \in E$ for all $m \in M$ then $(\rho, \sigma) \in E$. The last condition can be expressed: any span which is locally in $E$ is in $E$.

Notice that kernels are equivalence relations. (In fact, the condition of the last paragraph is satisfied with $V$ merely epic.)

Each relation $R$ on a family $u$ of objects generates, by an obvious countable process, an equivalence relation $E$ on $u$; moreover, a quotient for $R$ is precisely the same as a quotient for $E$.

If $A$ satisfies the conditions of Proposition 1 then each equivalence relation on $\left(u_{i}\right)_{i \in I}$ has the property: For each $i, j \in I$, there exists a monic $\operatorname{span}(\rho, \sigma) \in E_{j i}$ such that $E_{j i}$ is the crible generated by $(\rho, \sigma)$. 
To see this, take $(\rho, \sigma)$ to be the monic span obtained by applying Proposition 1(c) (with $M=2$ ) to the family of spans in $E_{j i}$.

An object $a$ of $A$ is called admissible when $A(a, b)$ is a small set for all objects $b$ of $A$. If $A$ has small homsets then all objects are admissible.

A congruence on $u=\left(u_{i}\right)_{i \in I}$ in $A$ is an equivalence relation $E$ on $u$ satisfying the following size condition: For each admissible object $a \in A$, there exists a small subset $S \subset I$ such that, for all $i \in I$ and $\alpha: a \rightarrow u_{i}$, there exists $j \in S$ and $\beta: a \rightarrow u_{j}$ with $(\alpha, \beta) \in E_{j i}$.

Of course, if $I$ is small, every equivalence relation on $u$ is a congruence. Any relation which satisfies the size condition generates a congruence.

PROPOSITION 6. Every kernel is a congruence.

Proof. Suppose $E$ is the kernel of $U=\left(\phi_{i}: u_{i} \rightarrow c\right)_{i \in I}$. If $a$ is admissible then $A(a, c)$ is small, so there exists a small set $S \subset I$ such that all arrows $a \rightarrow c$ which factor through some $\phi_{i}$ can be factored through a $\phi_{j}$ with $j \in S$. So $E$ is a congruence.

A category is called familially exact when it is familially regular and every congruence is a kernel.

THEOREM 7. Every congruence in a familially exact category has a quotient of which it is the kernel.

Proof. Let $E$ be a congruence on a family of objects. Then $E$ is the kernel of some family $U$. Apply Proposition 1(b) to $U$ to obtain $U=\mu_{*} V$ with $\mu$ monic and $V$ strong epic. Since $\mu$ is monic, $V$ has kernel $E$. Since $V$ is strong epic it is a quotient for $E$ (Theorem 3).

This leads us to define a category to be familially cocomplete when the conditions of Proposition 1 hold and every congruence has a quotient. Thus every familially exact category is familially cocomplete.

THEOREM 8. Familially cocomplete categories admit all small colimits.

Proof. If $I$ is small then the equivalence relation generated by the diagonal relation $\Delta$ on $u=\left(u_{i}\right)_{i \in I}$ is a congruence. A quotient for $\Delta$ gives the coproduct $\sum_{i \in I} u_{i}$.

To construct a coequalizer for $\alpha, \beta: a \rightarrow b$, let $E$ be the equivalence relation on the singleton family (b) generated by the single $\operatorname{span}(\alpha, \beta)$ from $b$ to $b$. A quotient for $E$ is a coequalizer for $\alpha, \beta$.

Notice that our use of "equivalence relation" basically agrees with the usual terminology for sets. An equivalence relation on a singleton family consisting of the set $x$ precisely amounts to an equivalence relation on $x$ in the usual sense; this is because the family of elements $1 \rightarrow x$ of $x$ is a strong epic family, so it is enough to say which elements of $x$ are related. In a general category, an equivalence relation on an object $a$ (as usually defined) amounts to a monic relation from $a$ to $a$ such that the crible of spans generated by it is an equivalence relation on the singleton family (a). 
THEOREM 9. The category $\mathscr{S}$ of small sets is familially exact.

Proof. Proposition 1(a) is well known. In $\mathscr{S}$, a family of arrows into $a$ is strong epic if and only if each element of $a$ is in the image of some member of the family; so it is clear that each $\alpha^{*}$ preserves strong epicness. Note that every object of $\mathscr{S}$ is admissible. It remains to see that every congruence $E$ on a family $\left(u_{i}\right)_{i \in I}$ is a kernel. Consider the equivalence relation on the set $\sum_{i \in I} u_{i}$ with elements $\xi: 1 \rightarrow u_{i}$, $\zeta$ : $1 \rightarrow u_{j}$ related when $(\xi, \zeta) \in E_{j i}$. The size condition on $E$ means that the set $c$ of equivalence classes is small. Then $E$ is the kernel of the family of functions $\lambda_{i}$ : $u_{i} \rightarrow c$ sending elements to their equivalence classes.

THEOREM 10. If $A$ is familially regular then so is any functor category $[X, A]$. When $X$ has small homsets, if $A$ is familially cocomplete or familially exact then so is $[X, A]$.

Proof. Proposition 1(a) for $A$ implies it for $[X, A]$, and so monics and strong epics are those which are pointwise thus. Hence, pullbacks of strong epic families in [ $X, A]$ are strong epic provided this is true in $A$.

Suppose $X$ has small homsets and $A$ is familially cocomplete. Suppose $E$ is a congruence on $g=\left(g_{i}\right)_{i \in I}$ in $[X, A]$. Let $\left(\rho_{i j}, \sigma_{i j}\right)$ be a monic span in $E_{j i}$ which generates $E_{j i}$ via the crible property. For each $x \in X$, let $E x$ be the saturated relation on $g x=\left(g_{i} x\right)_{i \in I}$ in $A$ generated by the monic spans $\left(\rho_{i j} x, \sigma_{i j} x\right)$ for each $i, j \in I$. Clearly, $E x$ is an equivalence relation since $E$ is. We claim $E x$ is a congruence. Take an admissible object $a \in A$. By Theorem 8 the copower $X(x,-) \cdot a$ exists. The Yoneda isomorphism

$$
[X, A](X(x,-) \cdot a, t) \cong A(a, t x)
$$

implies $X(x,-) \cdot a$ is admissible in $[X, A]$. Let $S \subset I$ be small as in the size condition for $E$ involving the admissible $X(x,-) \cdot a$; then $\alpha: a \rightarrow g_{i} x$ corresponds to $\rho$ : $X(x,-) \cdot a \rightarrow g_{i}$ for which there exists $j \in S$ and $\sigma: X(x,-) \cdot a \rightarrow g_{j}$ with $(\rho, \sigma) \in$ $E_{j i}$. Let $\beta: a \rightarrow g_{j} x$ correspond to $\sigma$. The span $(\alpha, \beta)$ has a span arrow into $(\rho x, \sigma x)$ induced by the coprojection $a \rightarrow X(x, x) \cdot a$. Since $(\rho x, \sigma x)$ is in $(E x)_{j i}$ so is $(\alpha, \beta)$. Thus $S$ is as in the size condition for $E x$ involving the admissible $a$.

Let $L x=\left(\lambda_{i} x: g_{i} x \rightarrow q x\right)_{i \in I}$ be a quotient for $E x$. By the universal property of quotients, we obtain a family $L=\left(\lambda_{i}: g_{i} \rightarrow q\right)_{i \in I}$ in $[X, A]$ which is a quotient for $E$. So $[X, A]$ is familially cocomplete.

Suppose $A$ is familially exact. We claim $E$ (as above) is the kernel of $L$. By Theorem 7,Ex is the kernel of $L x$; so the pullback span of $\lambda_{i} x, \lambda_{j} x$ is in $E x$ and hence has a span arrow into $\left(\rho_{i j} x, \sigma_{i j} x\right)$. Since the last span is monic, that span arrow is invertible. So $\left(\rho_{i j}, \sigma_{i j}\right)$ is a pointwise pullback for $\lambda_{i}, \lambda_{j}$. So the pullback of $\lambda_{i}, \lambda_{j}$ is in $E_{j i}$. So $E$ is the kernel of $L$.

THEOREM 11. Suppose $t: A \rightarrow X$ is a fully faithful functor with a left adjoint $s$. If $s$ is left exact then $X$ familially regular implies $A$ is. If $s$ preserves admissible objects then $X$ familially cocomplete implies $A$ is. If $s$ is left exact and preserves admissible objects then $X$ familially exact implies $A$ is. 
Proof. Straightforward.

A functor is said to have finite rank when it preserves filtered colimits. We write $\operatorname{Frank}(X, A)$ for the full subcategory of $[X, A]$ consisting of the functors of finite rank. An object $x$ of $X$ is called finitely presentable when it is admissible and $X(x,-)$ : $X \rightarrow \mathscr{S}$ has finite rank.

THEOREM 12. For all categories $X, \operatorname{Frank}(X, \mathscr{S})$ is familially regular. If every object of $X$ is a small filtered colimit of finitely presentable objects then $\operatorname{Frank}(X, \mathscr{S})$ is familially exact.

Proof. The inclusion $\operatorname{Frank}(X, \mathscr{S}) \rightarrow[X, \mathscr{S}]$ preserves arbitrary colimits and (since finite limits commute with filtered colimits in $\mathscr{S}$ ) finite limits. It follows that the inclusion preserves monics and strong epics. So $\operatorname{Frank}(X, \mathscr{S})$ is familially regular since $[X, A]$ is (Theorem 10 ).

Let $E$ be a congruence on $u=\left(u_{i}\right)_{i \in I}$ in $\operatorname{Frank}(X, \mathscr{S})$. Let $\left(\lambda_{i}: u_{i} \rightarrow c\right)_{i \in I}$ be a quotient for $E$ in the meta-category of all set-valued functors on $X$. Then $c$ has finite rank. The size condition on $E$ applied to the admissible $X(x,-)$, where $x$ is finitely presentable, yields that $c x$ is small for such $x$. Since every object of $X$ is assumed to be a small filtered colimit of finitely presentable objects and $c$ preserves filtered colimits, $c x$ is small for all $x$. The kernel for $\left(\lambda_{i}: u_{i} \rightarrow c\right)_{i \in I} \operatorname{in} \operatorname{Frank}(X, \mathscr{S})$ is clearly $E$. So $\operatorname{Frank}(X, \mathscr{S})$ is familially exact.

If $R$ is a relation on $u=\left(u_{i}\right)_{i \in I}$ in $A$ and $f: A \rightarrow X$ is a functor then we write $f R$ for the relation on $f u=\left(f u_{i}\right)_{i \in I}$ consisting of those spans $(f \rho, f \sigma)$ with $(\rho, \sigma)$ in $R$. If $R$ is saturated $f R$ is not in general. If $R$ is an equivalence relation, $f R$ is not in general, and neither is the saturation of $f R$. We say $f$ preserves the quotient of $R$ when it takes the quotient of $R$ to a quotient for $f R$.

THEOREM 13. Suppose $A$ is familially cocomplete and $f: A \rightarrow X$ preserves admissible objects and quotients of saturated reflexive symmetric transitive relations; then $f$ has $a$ right adjoint.

Proof. Take $x \in X$. Let $I$ be the set of pairs $(a, \xi)$ where $a \in A$ and $\xi$ : $f a \rightarrow x$ in $X$. Let $u=\left(u_{i}\right)_{i \in I}$ be the family with $u_{i}=a$ for $i=(a, \xi) \in I$. For $i=(a, \xi)$, $j=(b, \zeta) \in I$, let $R_{j i}$ be the set of spans $(\rho, \sigma)$ from $a$ to $b$ for which $\xi \cdot f \rho=\zeta \cdot f \sigma$. This $R$ is saturated reflexive symmetric and transitive. It also satisfies the size condition. For suppose $k$ is admissible in $A$. Then $f k$ is admissible so $X(f k, x)$ is small. So a small set of elements of $I$ will account for all composites $f k \stackrel{f \rho}{\rightarrow} f a \stackrel{\xi}{\rightarrow} x$. So the equivalence relation generated by $R$ is a congruence. Let $\left(\lambda_{i}: u_{i} \rightarrow c\right)_{i \in I}$ be a quotient for that congruence and so for $R$. Then $\left(f \lambda_{i}: f u_{i} \rightarrow f c\right)_{i \in I}$ is a quotient for $f R$. So there exists a unique $\gamma: f c \rightarrow x$ with $\xi=\gamma \cdot f \lambda_{i}$ for all $i=(a, \xi) \in I$. Then $\lambda_{(c, \gamma)}: c \rightarrow c$ is an idempotent on $c$ whose splitting gives the adjoint for $f$ at $x$.

4. Total and lex-total cocompleteness. Recall from $[8,6]$ that a category $A$ is [lex] total when is has small homsets and the Yondea embedding $y: A \rightarrow\left[A^{\mathrm{op}}, \mathscr{S}\right]$ has a [left-exact] left adjoint $z$. 
THEOREM 14. Suppose $A$ is a category with small homsets.

(a) $A$ is total if and only if it is familially cocomplete.

(b) $A$ is lex-total if and only if it is familially exact.

Proof. In each case "only if" follows from Theorems 9-11.

Suppose $A$ is familially cocomplete. Let $X$ be a replete full subcategory of $\left[A^{\text {op }}, \mathscr{S}\right]$ which contains the representables and has the left adjoint $z$ for $y$ defined on it. Let $X^{\prime}$ denote the full subcategory of $\left[A^{\mathrm{op}}, \mathscr{S}\right]$ consisting of those objects $t$ for which each pair of arrows $y a \rightarrow t, y b \rightarrow t$ has pullback in $X$.

We shall extend the definition of $z$ from $X$ to $X^{\prime}$. For each $t \in X^{\prime}$, choose a strong epic family $T=\left(\tau_{i}: y u_{i} \rightarrow t\right)_{i \in I}$ with each $u_{i} \in A$; for example, the family of all arrows from objects of the form ya. Let $R$ be the relation on $\left(u_{i}\right)_{i \in I}$ in $A$ for which $R_{j i}$ consists of the spans $(z \rho, z \sigma)$ from $u_{i}$ to $u_{j}$ where $\left(\rho: x \rightarrow y u_{i}, \sigma: x \rightarrow y u_{j}\right)$ is in the kernel of $T$ with $x \in X$. We are choosing $z$ to have $z y a=a$.

Let $\left(\pi_{i}: y u_{i} \rightarrow q\right)_{i \in I}$ be the quotient of $y R$ in the meta-category of set-valued functors on $A^{\text {op }}$. Since $T$ is strong epic it is the quotient of its kernel. Since $t \in X^{\prime}$, the kernel is generated by spans $(\rho, \sigma)$ with source $x$ in $X$. The unit $x \rightarrow y z x$ is an arrow of spans $(\rho, \sigma) \rightarrow(y z \rho, y z \sigma)$. So the kernel of $T$ is contained in the saturation of $y R$. So there exists a unique $\theta: t \rightarrow q$ such that $\theta \tau_{i}=\pi_{i}$ for all $i \in I$. Since $\left(\pi_{i}\right)_{i \in I}$ is epic, $\theta$ is epic. So $q$ lands in $\mathscr{S}$. So $\left(\pi_{i}\right)_{i \in I}$ is the quotient of $y R$ in $\left[A^{\mathrm{op}}, \mathscr{S}\right]$. Thus, for each $a \in A$, the following diagram exhibits $q a$ as a colimit in $\mathscr{S}$ :

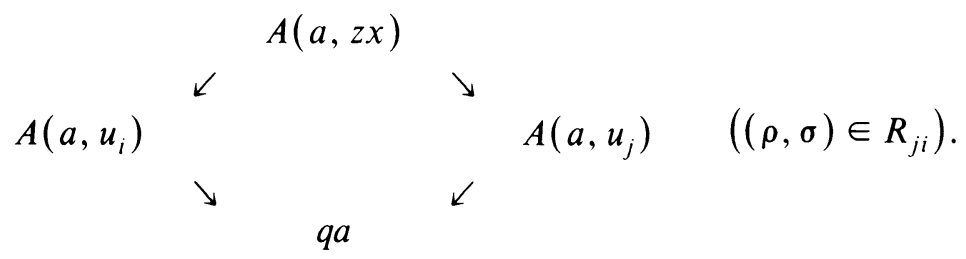

Let $S$ be a small subset of $I$ such that arrows $\sigma: a \rightarrow u_{j}$ with $J \in S$ yield all elements of $q a$. Thus the saturated reflexive symmetric transitive relation generated by $R$ satisfies the size condition. So the equivalence relation generated by $R$ is a congruence.

It follows that $R$ has a quotient $Z=\left(\zeta_{i}: u_{i} \rightarrow z t\right)_{i \in I}$ in $A$. To give an arrow $z t \rightarrow a$ is to give a family of arrows $u_{i} \rightarrow a$ whose kernel contains $R$. Since $t \in X^{\prime}$, this is to give a family of arrows $y u_{i} \rightarrow y a$ whose kernel contains the kernel of $T$. Since $T$ is strong epic, this is to give an arrow $t \rightarrow y a$. So we have extended $z$ to $X^{\prime}$.

To complete the proof of (a), begin with $X$ consisting of the representables in [ $A^{\text {op }}, \mathscr{S}$ ]. Certainly, $z$ is defined trivially on $X$. Hence $z$ extends to $X^{\prime}$. But $X^{\prime}$ contains all subobjects of representables since the pullback of $y a \rightarrow t, y b \rightarrow t$ is the pullback of $y a \rightarrow t \rightarrow y c, y b \rightarrow t \rightarrow y c$ for $t \rightarrow y c$ monic. Hence every object $t$ of $\left[A^{\mathrm{op}}, \mathscr{S}\right]$ is in $X^{\prime \prime}$ (since the pullback of $y a \rightarrow t, y b \rightarrow t$ has a monic into $y(a \times b)$ ). So $z$ extends to $\left[A^{\mathrm{op}}, \mathscr{S}\right.$ ]. So $A$ is total.

Suppose now that $A$ is familially exact. Take $X$ as before with the further restriction that it is closed under finite limits in $\left[A^{\mathrm{op}}, \mathscr{S}\right]$ and that $z$ is left exact on $X$. It is easy to see that $X^{\prime}$ is also closed under finite limits, so, in order to complete the proof of (b), it remains to show that the extension of $z$ to $X^{\prime}$ is also left exact. 
Since $A$ is familially regular, the equivalence relation $E$ generated by $R$ (in the construction of $z t$ above) has a simple description: $(\alpha, \beta) \in E_{j i}$ precisely when it is locally (in the sense of strong epic families) in the saturation of $R$.

This allows us to show that $z$ preserves pullbacks of the form:

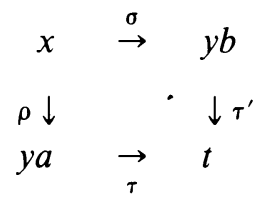

with $t \in X^{\prime}$. Since the $\operatorname{span}(\rho, \sigma)$ is in $X$ and $z$ is left exact on $X$, the $\operatorname{span}(z \rho, z \sigma)$ is monic. So it remains to show that each $\operatorname{span}(\alpha, \beta)$ with $z \tau \cdot \alpha=z \tau \cdot \beta$ has an arrow into $(z \rho, z \sigma)$. Choose $T$ for $t$ in such a way that $\tau, \tau^{\prime}$ are members. The equivalence relation $E$ generated by $R$ is the kernel of $Z$. So $(\alpha, \beta)$ is in $E$. So $(\alpha, \beta)$ is locally in the saturation of $R$. Since $(z \rho, z \sigma)$ is monic, it suffices to show that each $(\alpha, \beta)$ in the saturation of $R$ has an arrow into $(z \rho, z \sigma)$. But now we have $(\alpha, \beta) \rightarrow\left(z \rho^{\prime}, z \sigma^{\prime}\right)$ with $\left(\rho^{\prime}, \sigma^{\prime}\right)$ in the kernel of $T$. By the pullback property of $(\rho, \sigma)$ we obtain $\left(\rho^{\prime}, \sigma^{\prime}\right) \rightarrow(\rho, \sigma)$. Applying $z$ and composing, we obtain $(\alpha, \beta) \rightarrow(z \rho, z \sigma)$ as desired.

Suppose the following diagrams are pullback squares, the first in $X^{\prime}$, the second in A :

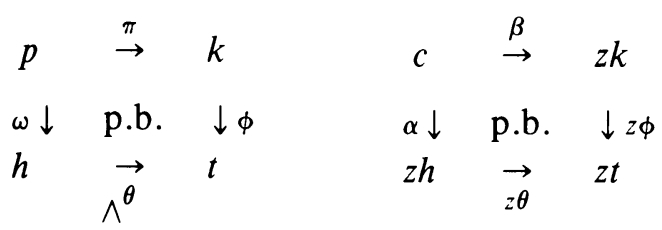

We shall show that the comparison arrow $\gamma: z p \rightarrow c$, with $\alpha \gamma=z \omega, \beta \gamma=z \pi$, is strong epic. Let $H, K$ be the families of all arrows from representables into $h, k$, respectively. For members $\alpha, \beta$ of $H, K$, respectively, consider the following diagram in which $x$ is the pullback of $\theta \alpha, \phi \beta$ :

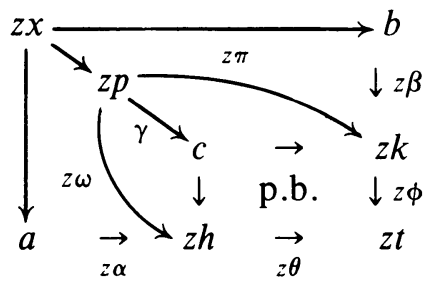

From the last paragraph, the outside is a pullback. Since $A$ is familially regular and $z H, z K$ are strong epic, the family of arrows $z x \rightarrow z p \stackrel{\gamma}{\rightarrow} c$ is strong epic. Hence $\gamma$ is strong epic as asserted.

For $S, t \in X^{\prime}$, we shall now prove $z(s \times t) \rightarrow z x \times z t$ is invertible. From the last paragraph, it is strong epic. It remains to prove it is monic. Take strong epic families $S=\left(\sigma_{m}: y v_{m} \rightarrow s\right)_{m \in M}, T=\left(\tau_{i}: y u_{i} \rightarrow t\right)_{i \in I}$ and let $x_{m n}, x_{i j} \in X$ be the pullbacks 
of $\left(\sigma_{m}, \sigma_{n}\right),\left(\tau_{i}, \tau_{j}\right)$, respectively. We have already seen that such pullbacks are preserved by $z$. A product of pullbacks is a pullback, so we have a pullback:

$$
\begin{array}{ccc}
z x_{m n} \times z x_{i j} & \rightarrow & v_{n} \times u_{j} \\
\downarrow & \text { p.b. } & \downarrow z \sigma_{n} \times z \tau_{j} \\
v_{m} \times u_{i} & \overrightarrow{z \sigma_{m} \times z \tau_{i}} & z s \times z t
\end{array}
$$

Since $x_{m n}, x_{i j} \in X$, we have $z\left(x_{m n} \times x_{i j}\right) \cong z x_{m n} \times z x_{i j}$; and the product of the pullbacks $x_{m n}, x_{i j}$ is preserved by $z$. So we also have the pullback:

$$
\begin{array}{ccc}
z x_{m n} \times z x_{i j} & \rightarrow & v_{n} \times u_{j} \\
\downarrow & \text { p.b. } & \downarrow \\
v_{m} \times u_{i} & \rightarrow & z(s \times t)
\end{array}
$$

Consequently, the families

$$
\left(v_{m} \times u_{i} \rightarrow z(s \times t)\right)_{m \in M, i \in I} \text { and }\left(v_{m} \times u_{i} \rightarrow z s \times z t\right)_{i \in I, m \in M}
$$

have the same kernel. By Proposition $2, z(s \times t) \rightarrow z s \times z t$ is monic.

To complete the proof that $z$ is left exact on $X^{\prime}$ it remains to show that $z$ preserves monics in $X^{\prime}$. Suppose $\mu: s \rightarrow t$ is a monic in $X^{\prime}$. Let $T=\left(\tau_{i}: y u_{i} \rightarrow t\right)_{i \in I}$ be strong epic and form the pullbacks:

$$
\begin{array}{ccc}
s_{i} & \stackrel{\sigma_{i}}{\rightarrow} & s \\
\mu_{i} \downarrow & \text { p.b. } & \downarrow \mu \\
y u_{i} & \overrightarrow{\tau_{i}} & t
\end{array}
$$

Then $s_{i} \in X^{\prime}$ and $\left(\sigma_{i}\right)_{i \in I}$ is strong epic. Let $x_{i j} \in X$ be the pullback of $\tau_{i}, \tau_{j}$. Let $p_{i j}$ be the pullback of $\sigma_{i}, \sigma_{j}$; so $s$ is the quotient of the relation consisting of these pullback spans (Theorem 3). Notice that, since $\mu$ is monic, $p_{i j}$ is the limit of the diagram indicated below.

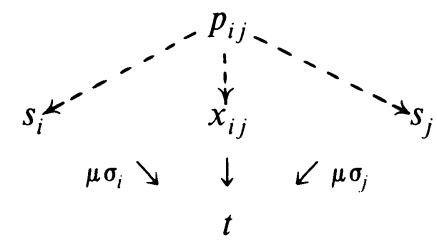

We have the following properties:

(i) $z s$ is the quotient of the relation consisting of the spans $z p_{i j}$ from $z s_{i}$ to $z s_{j}$ (since $z$ is a left adjoint);

(ii) $z x_{i j}$ is the pullback of $z \tau_{i}, z \tau_{j}$ (as seen earlier);

(iii) the comparison arrow from $z p_{i j}$ to the limit $l_{i j}$ of the following diagram is a strong epic (since $\gamma$ is strong epic):

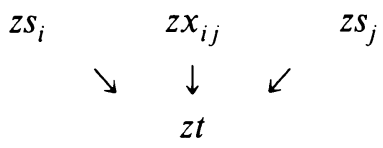


These properties allow us to prove $z \mu$ is monic as follows. Take $\xi, \zeta: c \rightarrow z s$ with $(z \mu) \xi=(z \mu) \zeta$. Form the limit

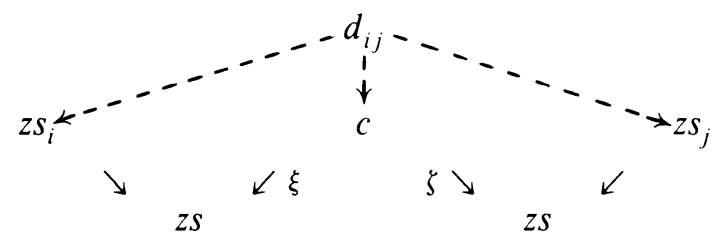

to obtain a strong epic family of arrows $d_{i j} \rightarrow c$ (using familial regularity). Since $(z \mu) \xi=(z \mu) \zeta$, we obtain an arrow $d_{i j} \rightarrow z x_{i j}$, and hence an arrow $d_{i j} \rightarrow l_{i j}$. The pullback of the strong epic $z p_{i j} \rightarrow l_{i j}$ along this last arrow gives a strong epic $\bar{d}_{i j} \rightarrow d_{i j}$ such that $\bar{d}_{i j} \rightarrow d_{i j} \rightarrow c$ equalizes $\xi, \zeta$. So $\xi=\zeta$. So $z \mu$ is monic.

5. Toposes. A topology [1] on a category $X$ is a function which assigns to each object $x$ of $X$ a set of families $\Gamma$ of arrows into $x$ (each such family $\Gamma$ is called a cover of $x$ ) such that the following conditions are satisfied:

T1. The singleton family consisting of the identity of $x$ is a cover of $x$.

T2. If $\Gamma$ is a cover of $x$ and $\rho: y \rightarrow x$ is any arrow then $\rho^{*} \Gamma$ is isomorphic to a cover of $y$.

T3. The composite of covers is isomorphic to a cover.

(The concepts needed for T2, T3 were defined in \$1.)

A functor $f: X^{\text {op }} \rightarrow A$ is called a sheaf for the topology on $X$ when, for all objects $x$ of $X$ and all covers $\Gamma$ of $x$, the functor $f: X \rightarrow A^{\text {op }}$ takes $\Gamma$ to a quotient for $f$ of the kernel of $\Gamma$.

THEOREM 15. The full subcategory $\operatorname{Sh}(X, \mathscr{S})$ of $\left[X^{\mathrm{op}}, \mathscr{S}\right]$, consisting of the sheaves for a given topology on $X$, is familially regular.

Proof. The inclusion $\operatorname{Sh}(X, \mathscr{S}) \rightarrow\left[X^{\text {op }}, \mathscr{S}\right]$ creates all limits which exist in $\left[X^{\mathrm{op}}, \mathscr{S}\right]$. So $\operatorname{Sh}(X, \mathscr{S})$ satisfies Proposition 1(a).

A monic $\mu: p \rightarrow f$ in $\left[X^{\mathrm{op}}, \mathscr{S}\right]$ is called dense when, for all $\alpha \in f x$, there exists a cover $\Gamma$ of $x$ such that, for each member $\xi: x^{\prime} \rightarrow x$ of $\Gamma$, the element $(f \xi) \alpha$ is in the image of the function $\mu x^{\prime}: p x^{\prime} \rightarrow f x^{\prime}$.

We claim that a family $U=\left(\phi_{i}: u_{i} \rightarrow f\right)_{i \in I}$ is strong epic in $\operatorname{Sh}(X, \mathscr{S})$ if and only if $U=\mu_{*} V$ in $\left[X^{\mathrm{op}}, \mathscr{S}\right]$ where $\mu$ is a dense monic and $V$ is (strong) epic. To prove "if", suppose $U=\mu_{*} V$ as asserted. If $U=\nu_{*} W$ where $\nu: t \rightarrow f$ is monic and $t$ is a sheaf then $\mu$ factors through $\nu$ since $V$ is strong epic. So $\nu$ is a dense monic between sheaves. Clearly then each $\nu x: t x \rightarrow f x$ is a retraction. So $\nu$ is invertible. So $U$ is strong epic in $\operatorname{Sh}(X, \mathscr{S})$.

Conversely, suppose $U$ is strong in $\operatorname{Sh}(X, \mathscr{S})$. Let $U=\mu_{*} V$ be the factorization of $U$ into a monic $\mu$ and strong epic family $V$ in $\left[X^{\mathrm{op}}, \mathscr{S}\right]$. We must show that $\mu$ : $p \rightarrow f$ is dense. For each $x \in X$, put $p^{+} x$ equal to the set of $\alpha \in f x$ for which there is a cover $\Gamma$ of $x$ with $(f \xi) \alpha$ in the image of $\mu x^{\prime}$ for all members $\xi: x^{\prime} \rightarrow x$ of $\Gamma$. By T2, $p^{+}$is a subfunctor of $f$. By T3, $p^{+}$is a sheaf. So $\mu$ factors through the inclusion $\nu$ : $p^{+} \rightarrow f$. Since $U$ is strong epic in $\operatorname{Sh}(X, \mathscr{S}), \nu$ is invertible. So $p^{+}=f$ which clearly means $\mu$ is dense. 
Since a pullback of a dense monic is clearly a dense monic, it follows that $\gamma^{*}$ takes strong epic families for all arrows $\gamma$ in $\operatorname{Sh}(X, \mathscr{S})$.

REMARKS. (1) The existence of a left-exact left adjoint to $\operatorname{Sh}(X, \mathscr{S}) \rightarrow\left[X^{\mathrm{op}}, \mathscr{S}\right]$ (the "associated sheaf" [1]) implies that $\operatorname{Sh}(X, \mathscr{S})$ is familially exact when $X$ has small homsets and there exists a small set $G$ of objects $X$ such that, for all objects $x$ of $X$, there exists a cover of $x$ whose members all have sources in $G$.

(2) If $A$ is familially regular then the strong epic families are the covers for a topology on $A$. This is the largest topology for which the representable functors are sheaves (Corollary 4). By Theorem $15, \operatorname{Sh}(A, \mathscr{S})$ is familially regular. If $A$ has small homsets, the Yoneda embedding yields a fully faithful functor $A \rightarrow \operatorname{Sh}(A, \mathscr{S})$ which preserves all limits and strong epic families. If $A$ has a small cogenerator then (by Remark (1)) $\operatorname{Sh}(A, \mathscr{S})$ is familially exact and provides the "familial exactification" of the familially regular $A$.

(3) Familial exactification will be studied elsewhere (for more general $A$ than in (2)), however, the ideas of [4] are closely related and led to unpublished work of A. Carboni and R. Walters which uses enriched categories to provide free constructions. Notice in (2) that $\operatorname{Sh}(A, \mathscr{S})$ coincides with the usual categorical construction on $A$ which cocompletes $A$ with a view to preserving quotients of kernels. The reason this does not work when $A$ does not have a small cogenerator is that the colimits which need to be added (to gain quotients of congruences) are not all small.

THEOREM 16. (a) Familial regularity implies regularity and the existence of a strict initial object.

(b) Familial exactness implies exactness and the existence of small disjoint universal coproducts.

(c) For a category with small homsets, a small strong generator and small coproducts, if the coprojection into any small coproduct pullback along any arrow into the coproduct to a strong epic family then the converse of (a) holds.

(d) For a category with small homsets and a small strong generator, the converse of (b) holds.

Proof. (a) Assume familial regularity. Proposition 1(a) gives finite limits, Proposition 1(b) with $U$ a singleton family gives factorizations of arrows into a strong epic arrow followed by a monic. Since $\alpha^{*}$ preserves strong epicness of families, pullback along $\alpha$ takes strong epic arrows to strong epic arrows. So we have regularity.

The empty family $U$ into 1 factors as $U=\mu_{*} V$ where $\mu: c \rightarrow 1$ is monic and $V$ is strong epic. Since $U$ is empty, $V$ is empty. By Theorem $3, V$ is the quotient of its kernel; so $c$ is initial. For any $\alpha: a \rightarrow c$, the family $\alpha^{*} V$ is strong epic into $a$. Since $\alpha^{*} V$ is empty, $a$ too is initial. So any $\alpha: a \rightarrow c$ is invertible. So $c$ gives a strictly initial object 0 .

(b) Assume familial exactness. An equivalence relation on $a$ has its saturation a congruence and hence a kernel of some arrow $\tau: a \rightarrow b$. The pullback $(\rho, \sigma)$ of $\tau$ with itself is in the kernel of $\tau$. Since the equivalence relation is monic and has $(\rho, \sigma)$ in its saturation, $(\rho, \sigma)$ is isomorphic to the equivalence relation which is thus a pullback for $\tau, \tau$. 
Since we have familial regularity, we have a strict initial object 0 , by (a). Let $\left(u_{i}\right)_{i \in I}$ be a family of objects with $I$ small. The congruence generated by the diagonal relation $\Delta$ merely adds spans with initial sources to the spans of $\Delta$. To say this congruence is the kernel of its quotient is to say the quotient is a coproduct for $\left(u_{i}\right)_{i \in I}$ and that this coproduct is disjoint (as usual, disjointness includes monicness of the coprojections). If $\alpha: a \rightarrow c$ is an arrow into the coproduct $c$ of $\left(u_{i}\right)_{i \in I}$ then $\alpha^{*}$ takes the family of coprojections into a strong epic family into $a$ whose kernel is the congruence generated by the diagonal relation on the family of pullback objects $a \times{ }_{c} u_{i}$; so $a$ is the coproduct of the objects. So small coproducts are universal.

(c) Assume the hypotheses of (c). Regularity implies finite limits exist. Let $U=\left(\phi_{i}: u_{i} \rightarrow a\right)_{i \in I}$ be any family of arrows into $a$. For each $i$, there is a small strong epic family $V_{i}$ whose members all have source in $G$. The composite family $U V$ has only a small set of members since $G$ is small and the category has small homsets. Let $c$ be a coproduct for the family of sources of these members indexed by the set of members. The members induce an arrow $\gamma: c \rightarrow a$ which, by regularity, factors as $\gamma=\mu \varepsilon$ where $\mu: m \rightarrow a$ is monic and $\varepsilon$ is strong epic. So $U V=\mu_{*} W$ where $W$ is the strong epic family of coprojections into $c$ composed with $\varepsilon$. Since each $V_{i}$ is strong epic and $\mu$ is monic, $U=\mu_{*} W^{\prime}$ for some family $W^{\prime}$. Then $W=W^{\prime} V$, so $W^{\prime}$ is strong epic. So Proposition 1(b) holds.

Suppose now that $V$ (as above) is a strong epic family and proceed as above to obtain $\gamma: c \rightarrow a$ which is strong epic since $U V$ is. For any arrow $\alpha: b \rightarrow a$, the pullback $\gamma^{\prime}: c^{\prime} \rightarrow b$ of $\gamma$ along $\alpha$ is strong epic (by regularity). By hypothesis, the pullbacks of the coprojections into $c$ along $c^{\prime} \rightarrow c$ are a strong epic family. Since $\gamma^{\prime}$ composed with these pullbacks gives a strong epic family $\leqslant \alpha^{*} U$, we have that $\alpha^{*} U$ is strong epic. So the category is familially regular.

(d) Assume the hypotheses of (d). Then we have familial regularity by (c). Let $E$ be a congruence on $\left(u_{i}\right)_{i \in I}$.

First consider the case where $I$ is small. Let $\left(\rho_{j i}, \sigma_{j i}\right)$ be the monic span generating the crible $E_{j i}$ and let $r_{j i}$ be the source of $\rho_{j i}, \sigma_{j i}$. Let $\tau, v$ be the arrows $\sum_{i, j \in I} r_{j i} \rightarrow$ $\sum_{i \in I} u_{i}$ induced by the $\rho_{j i}, \sigma_{j i}$, respectively, for $i, j \in I$. Universality of small coproducts implies that $(\tau, v)$ is a monic span from $\sum u_{i}$ to itself. Since $E$ is an equivalence relation and small coproducts are disjoint, $(\tau, v)$ is an equivalence relation on the object $\sum u_{i}$. So there exists $\lambda: \sum u_{i} \rightarrow c$ such that $(\tau, v)$ is the pullback of $\lambda, \lambda$. Thus we obtain $\lambda_{i}: u_{i} \rightarrow c$ for all $i \in I$. Disjoint universality of small coproducts yields that $\left(\rho_{j i}, \sigma_{j i}\right)$ is the pullback of $\lambda_{i}, \lambda_{j}$. So $E$ is the kernel of $\left(\lambda_{i}\right)_{i \in I}$.

Now consider $I$ arbitrary. Since all objects are admissible and $G$ is small, there exists a small subset $S$ of $I$ such that, for all $\rho: g \rightarrow u_{i}$ with $g \in G$, there exists $j \in S$ and $\sigma: g \rightarrow u_{j}$ with $(\rho, \sigma) \in E_{j i}$. Restricting $E$ to $\left(u_{i}\right)_{i \in S}$, we obtain $\left(\lambda_{i}: u_{i} \rightarrow c\right)_{i \in S}$ for which the restricted $E$ is a kernel (by the above "small" case). For each $i \in I$, define $\Theta_{g}: A\left(g, u_{i}\right) \rightarrow A(g, c)$ by $\Theta_{g}(\rho)=\lambda_{j} \sigma$ where $j \in S,(\rho, \sigma) \in E_{j i}$. Since $E$ is an equivalence relation this is independent of the choice of $j, \sigma$, and $\Theta_{g}$ is natural in $g$. By Corollary 5, $G$ is dense; so there exists a unique $\lambda_{i}: u_{i} \rightarrow c$ with $\Theta_{g}(\rho)=\lambda_{i} \rho$ for all $\rho$. Clearly, $E$ is the kernel of $\left(\lambda_{i}\right)_{i \in I}$. 
Theorems 14 and 15 have the corollary that an exact category with small homsets, a small strong generator, and small disjoint universal coproducts is lex-total. Of course this is an immediate consequence of Giraud's Theorem [1, p. 303]; however, the present format seems more tractable when dealing with enriched categories as in the current work of Brian Day and the author.

\section{REFERENCES}

1. M. Artin, A Grothendieck and J. L. Verdier (eds.), Theorie des topos et cohomologie etale des schemas, Lecture Notes in Math., vol. 269, Springer-Verlag, Berlin and New York, 1972.

2. M. Barr, Exact categories, Lecture Notes in Math., vol. 236, Springer-Verlag, Berlin and New York, 1971, pp. 1-120.

3. R. Betti, A. Carboni, R. Street and R. Walters, Variation through enrichment, J. Pure Appl. Algebra (to appear).

4. A. Carboni and R. Celia Magno, The free exact category on a left exact one, J. Austral. Math. Soc. Ser. A 33 (1982), 295-301.

5. G. M. Kelly, Monomorphisms, epimorphisms and pullbacks, J. Austral. Math. Soc. 9 (1969), 124-142.

6. R. Street, Notions of topos, Bull. Austral. Math. Soc. 23 (1981), 199-208.

7. Cauchy characterization of enriched categories, Rend. Sem. Mat. Fis. Milano 51 (1981), 217-233.

8. R. Street and R. Walters, Yoneda structures on 2-categores, J. Algebra 50 (1978), 350-379.

9. $\mathrm{R}$. Succi-Cruciani, La theoria delle relazione nelle studio di categorie regolarie di categorie esatte, Riv. Mat. Univ. Parma (4) 4 (1975), 143-158.

10. W. Tholen, Note on total categories, Bull. Austral. Math. Soc. 21 (1980), 169-174.

11. R. F. C. Walters, Sheaves on sites as cauchy-complete categories, J. Pure Appl. Algebra 24 (1982), 95-102.

12. R. Wood, Some remarks on total categories, J. Algebra 75 (1982), 538-545.

School of Mathematics and Physics, Macquarie University, North Ryde, New South Wales 2113, Australia 